\title{
Data-adaptive filtering and the state of the art in image processing (Presentation Video)
}

\section{Peyman Milanfar}

Peyman Milanfar, "Data-adaptive filtering and the state of the art in image processing (Presentation Video)," Proc. SPIE 9216, Optics and Photonics for Information Processing VIII, 92160Q (19 September 2014); doi:

$10.1117 / 12.2063108$

SPIE Event: SPIE Optical Engineering + Applications, 2014, San Diego, California, United States 


\title{
Data-adaptive filtering and the state of the art in image processing (Presentation Video)
}

\author{
Peyman Milanfar, Univ. of California, Santa Cruz (United States)
}

\begin{abstract}
The most effective recent approaches to processing and restoration of images and video are ones that flexibly adapt themselves to the content of these signals. These high performance methods have come about through the convergence of several powerful ideas from different science and engineering disciplines. Examples include Moving Least Square (from computer graphics), the Bilateral Filter and Anisotropic Diffusion (from computer vision), Boosting and Spectral Methods (from Machine Learning), Non-local Means and Bregman Iterations (from Applied Math), Kernel Regression and Iterative Scaling (from Statistics). These approaches are deeply connected; and in this talk, I will present a framework for understanding many common underpinnings of these ideas. This has led us to new insights and algorithms, yielding both deeper theoretical analysis, and state of the art results in practice.
\end{abstract}

View presentation video on SPIE's Digital Library: http://dx.doi.org/10.1117/12.2063108 